\title{
Dynamic behavior and failure of the base and heat affected materials of a HSS fillet welded joint
}

\author{
Julien Carrier ${ }^{1}$, a, Eric Markiewicz ${ }^{1}$, Grégory Haugou ${ }^{1}$, David Lebaillif ${ }^{2}$, Nicolas Leconte ${ }^{1}$, and Hakim Naceur ${ }^{1}$ \\ ${ }^{1}$ Université de Valenciennes, LAMIH CNRS 8201, Le Mont-Houy, CISIT, 59313 Valenciennes Cedex 9, France \\ ${ }^{2}$ Nexter Systems, 11 allée des Marronniers, 78022 Versailles, France
}

\begin{abstract}
Welded joints, due to their manufacturing process, are commonly weakened areas. This study analyses the dynamic behavior of the Base Metal (BM) and the Heat-Affected Zone (HAZ) materials of a HSS (High Strength Steel) fillet welded joint. First, a specific approach is developed to generate the HAZ material using a thermal treatment. Hardness and grain size are used to validate the replicated HAZ. This approach appears efficient and repeatable. Secondly, the true stress-strain quasi-static and dynamic behaviors up to failure of the BM and the HAZ are determined. This characterization is performed thanks to video tracking procedure and Bridgman-LeRoy correction. The comparison between these two materials shows that the thermal field of the welding process increases the HAZ yield stress and hardening while decreasing the strain at failure. It appears that the base metal is not rate sensitive from quasi-static up to $1350 \mathrm{~s}^{-1}$. On the contrary, the heat affected material appears to be rate sensitive but by softening. This unexpected dynamic material softening requires further analyses.
\end{abstract}

\section{Introduction}

Finite element modeling is currently used to model the dynamic behavior of structures submitted to blast events. It allows to design armoured vehicles by evaluating the strength and integrity of the structure against battlefield threats. Welded joints, due to their manufacturing process, are weakened areas. Since it is difficult to model the failure of welded joints in a coarse model, a meso-scale model is proposed. So, mechanical properties up to failure of the constitutive materials: Base Metal (BM), HeatAffected Zone (HAZ) and Weld Metal (WM) have to be supplied. In this paper, only the BM and the HAZ are presented. Multi-scale approaches have been already successfully applied on a mild-steel spot-welded assembly ([1] \& [2]) or on aluminum welded joint [3]. A similar method is investigated for a HSS fillet welds assembly subjected to dynamic loadings. Several studies about the mechanical strength of welded assemblies published in the open literature cover a large field of loadings: fatigue [4], quasi-static [5] and low velocity loadings [6]. However, finite element models of military applications are not well expanded in literature. Gaudreault [7] is almost the only one who presented a study dedicated to a welded HSS joint subjected to an explosion. The welded joint is modeled by a shell element with the HAZ materials properties. The weld geometry is not considered so the stress concentration (and the fracture behavior) could not be really predictive. Few papers reported the HSS material behavior at high strain rate used for armoured vehicles ([8] \& [9]), even less under a tensile loading as well as the dynamic behavior of welded assembly manufactured with these specific steels. Besides, open literature does not

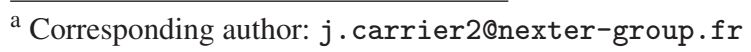

cover mechanical strength of the associated HAZ material. In this paper, a specific approach is presented to replicate the HAZ material using a thermal treatment. Then, quasistatic and dynamic behavior up to failure of the BM and the HAZ are compared and analyzed.

\section{Experimental program}

The manufacturing process and tensile specimen geometries, as well as experimental tests, measurement facilities and analysis procedure are presented in this section.

\subsection{Materials}

The BM is a hot-rolled HSS that offers high strength, ductility and good weldability. Due to the small size of the HAZ, dumbbell tensile specimens cannot be extracted directly from the welded joint to perform quasi-static and dynamic tests. As a consequence, a specific approach is developed to generate the HAZ material using a thermal treatment. Both hardness and grain size are controlled to validate the replicated HAZ with respect to the real one. Grain size is measured by the grain size number ( $G$ which is an integer) through the following relation (defined by the norm):

$$
M=8.2^{G}
$$

where $M$ is the number of grain per $\mathrm{mm}^{2}$ on the observed sample surface. The Vickers hardness is measured along 6 paths across the BM, HAZ and WM. As seen in Fig. 1, the hardness between BM and HAZ increase significantly.

The hardness in the heat affected material doesn't remain constant along the path. The question is: how to take into account the gradient of properties? Our choice is to determine the extreme characteristics of the HAZ: on one hand it's given by the BM and, on the other hand, by 


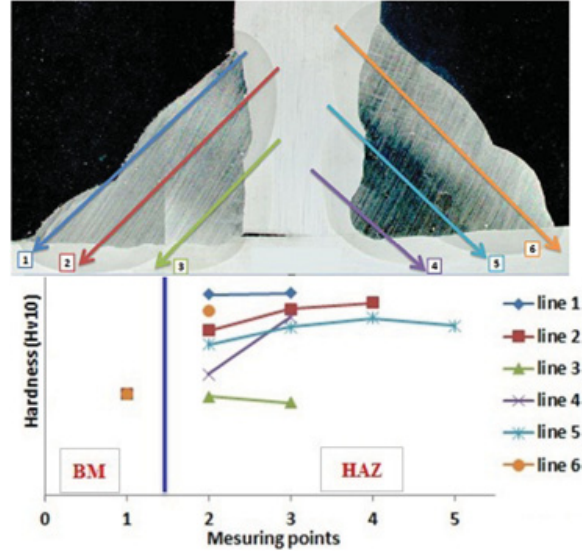

Figure 1. Hardness specimen and hardness measurement in the $\mathrm{BM}$ and HAZ materials.

the maximum hardness zone in the HAZ. This hardness is then considered for the heat treatment. Furthermore, this maximum hardness is located where the maximum deformation will occur under dynamic loadings due to high geometrical stress concentration. The aim of this heat treatment is not to re-create the real and complex thermal cycle induced by the welding process (i.e. by using a Gleeble $^{\circledR}$ thermomechanical simulator), but only to obtain specimens with mechanical properties close to the real HAZ.

The heat treatment is detailed below. The sample is put in a preheat furnace. The temperature is risen to a specific temperature (austenitic temperature - Tc) and remains constant during several minutes. The sample is then tempered to fix the microstructure. To adjust the final hardness of the sample a low temperature stress-relieving treatment is done.

A preliminary study was carried out to adjust the temperature $(\mathrm{Tc})$ to obtain the correct austenite grain size in the material before the tempering (Fig. 2a). The first temperature tested was too low to completely remove ferrite and pearlite in the material. As the austenitic temperature raises, the hardness and the grain size number decrease.

The HAZ microstructure and the hardness are finally validated by comparison with the real one (obtained in the welded joint) (Fig. 2).

To avoid excessive distortions of the specimen, the thermal cycle is applied on the rough sample, and then the final geometry is machined.

The heat treatment process is applied on 5 samples per batch: 4 samples will be machined at the tensile specimen geometry; the last one is the batch control specimen. This one is cut in the middle to make hardness measurements on the position corresponding to the gage cross section (Fig. 3). 6 batches of 5 samples are made. Hardness measurements remain constant for all batches: less than $2 \%$ of dispersion is measured.

\subsection{Specimen geometries}

Quasi-static and dynamic tests are performed on smooth axisymmetric specimens at room temperature. As shown in Fig. 4, specimen geometries with different gage lengths

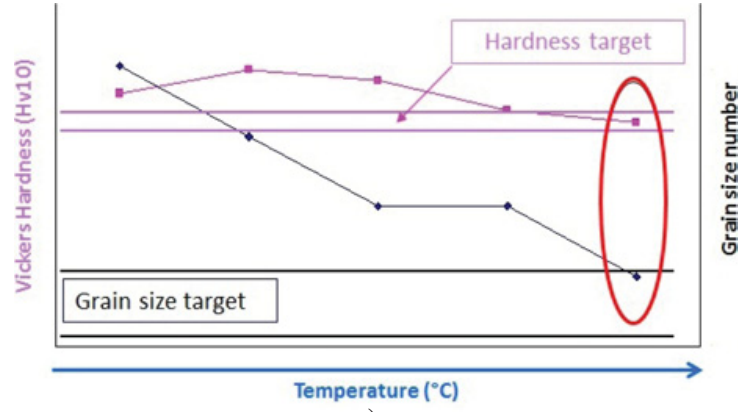

a)

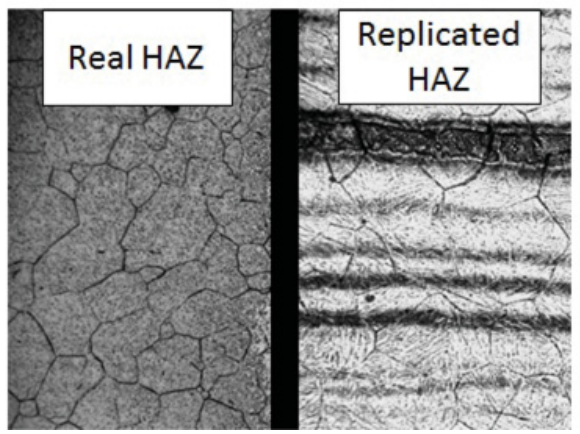

b)

Figure 2. a) Description of the optimized heat treatment; b) comparison of real and replicated HAZ microstructure.

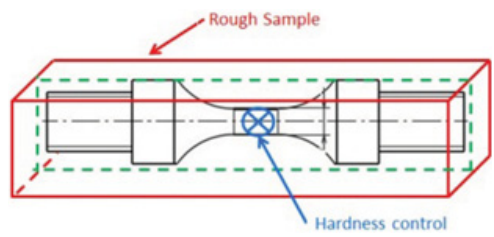

Figure 3. Rough sample.

and diameters are required to cover a large range of strain rates from quasi-static to $1350 \mathrm{~s}^{-1}$. Quasi-static geometry complies the standards (Fig. 4a). Different dynamic geometries are considered in order to compare $\mathrm{BM}$ and HAZ materials properties at the same strain rate. Gage diameters have been adapted to perform dynamic tests with the same pre-loading force on a pre-stretched tension bar. The strain rates are measured in the gage length of the sample during plasticity before necking and after the wave stabilization. All tests are performed 3 times for quasi-static tests and 5 times for dynamic tests.

\subsection{Pre-stretched tension bar}

Experimental program based on dynamic loadings is carried out using Hopkinson bars technique. As the high ductility properties of the base material and heat affected zones have been observed under quasi static conditions, a high duration time is wished. For that, as shown by Fig. 5, a pre-stretched bar device, based on the original concept proposed by Albertini [10], is used to determine the set of flow curves for moderate strain rates up to failure. Both bars have a common diameter equal to $11 \mathrm{~mm}$ and the specimen is inserted between positions $\mathrm{D}$ and $\mathrm{E}$. The pre-loading force $\mathrm{F}$ - stabilized between $\mathrm{A}$ and $\mathrm{B}$ - is 


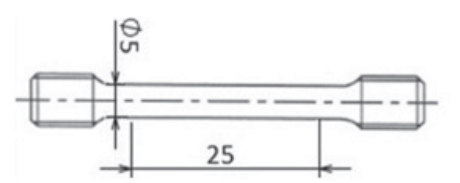

a) BM-QS \& HAZ-QS

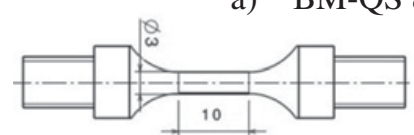

b) BM-1

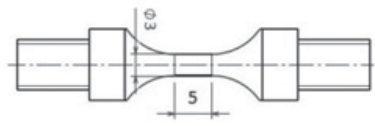

c) $\mathrm{BM}-2$

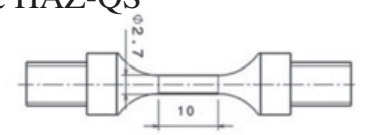

d) HAZ-1

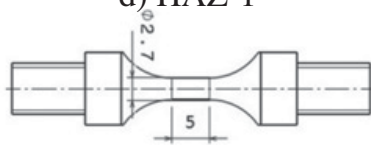

e) HAZ-2
Figure 4. Geometries of tensile specimens: a) quasi-static for BM and HAZ; b) c) dynamic for BM; d) e) dynamic for HAZ.

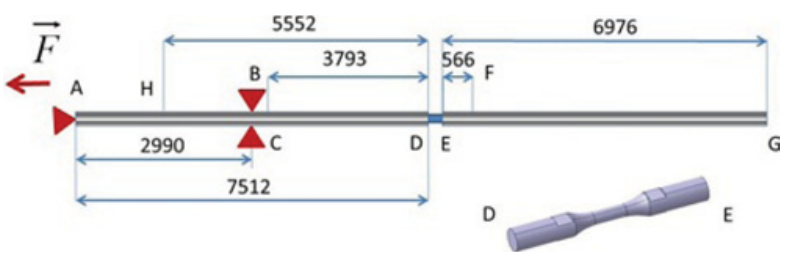

Figure 5. Sketch of the pre-stretched tension bar - Dimensions in $\mathrm{mm}$.

provided by two quasi-static loadings applied on the input bar (Fig. 5).

This ensures the storage of an elastic energy brutally released when the brittle fracture of the fuse occurs at the position B. The elastic wave propagates with a constant duration time depending on the length of the pre-stretched part $(\mathrm{AB})$ along the input bar and loads the specimen up to failure. A set of three full strain bridges HBM LY $41350 \Omega-1.5 \mathrm{~mm}$ in length) is strategically positioned along the experimental set-up to detect the elastic waves system using a high speed recorder (Yokogawa DL 750 4 channels). The data are finally recorded to compute the stress/strain curves [11]. Here, the position in B is chosen so that no superposition of the reflected wave with the incident one can occur at the position of the strain gage bonded in $\mathrm{C}$. The strain rate value is changed by varying the pre-loading force $\mathrm{F}$ of the pre-stretched device.

\subsection{Measurement of specimen contour evolution by video tracking}

Based on previously published works ([12] \& [13]), a methodology is set up to measure the specimen contour evolution after necking to obtain the true stress-strain curve of the material. A CMOS high speed camera (Photron APX RS 3000) is added to record the shape evolution of the specimen during the test. As sharp edges between the background and the specimen are needed, a flashlight is placed behind the specimen so as to highlight its shadow.

Then, the elongation and reduction of the specimen can be quantified using a Matlab ${ }^{\circledR}$ routine. Figure 6 provides details on the optical measurement device and the image analysis progress. The speed of the camera is set to 75000

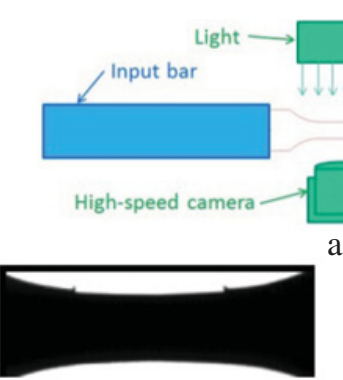

b)

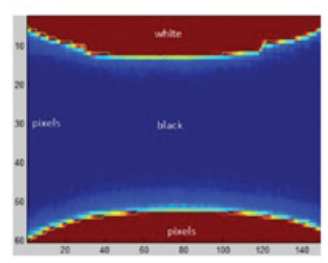

d)

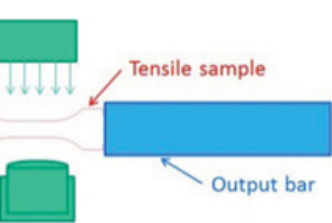

a)

c)

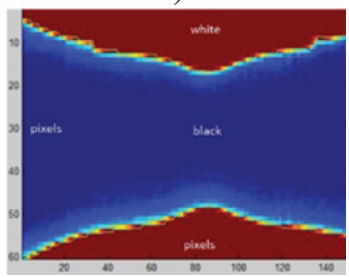

e)

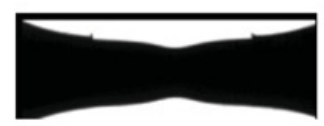

Figure 6. a) Optical measurement device; b) c) Initial images at the start of test and during test; d) e) Images after filtering procedure.

and $90000 \mathrm{f} / \mathrm{s}$ depending on the range of strain rates so as to ensure over 20 images during the test.

Diameter reduction measurement, in particular the smallest diameter after necking, is obtained by a tracking algorithm with a filter on the contrast gradient. The minimum diameter is the minimum distance between the two filtered edges of the specimen. The first image, just before the test starts, is used to calibrate the original gage diameter of the specimen (in pixels) (Fig. $6 b \& d$ ). The final diameter measured by video tracking (last diameter at fracture initiation) is compared to the post-mortem diameter to validate the digital calibration. The true stressstrain curve is obtained using the following relations:

$$
\varepsilon_{\text {true }}=2 \ln \left(D_{0}\right)-2 \ln (D)
$$

where $D_{0}$ is the initial gage diameter and $D$ is the minimum diameter in the necked area.

$$
\sigma_{\text {true }}=\sigma_{z z}=\frac{4 F}{\pi \cdot D^{2}}
$$

where $F$ is the force measured during the test.

The high speed camera is limited to a picture resolution of $64 \times 250$ pixels on the specimen gage section. This low resolution and the limited number of recorded images (about 20) lead to an error of about $0.05 \mathrm{~mm}$ on the measured diameter. Therefore, after the onset of necking, an error of about $50 \mathrm{MPa}$ on the true stress and 0.03 on the true strain is estimated.

\subsection{Bridgman-LeRoy correction}

True stress-true strain diagram cannot be computed beyond necking, unless the effects of necking on the geometry of the tensile specimen and the stress state are accurately quantified. Necking produces a triaxial stress state that does not reflect the true uniaxial flow stress of the material. Therefore, the true uniaxial stress $\left(\sigma_{z z}\right)$ must be multiplied by a correction factor to correct the effect of the triaxial 


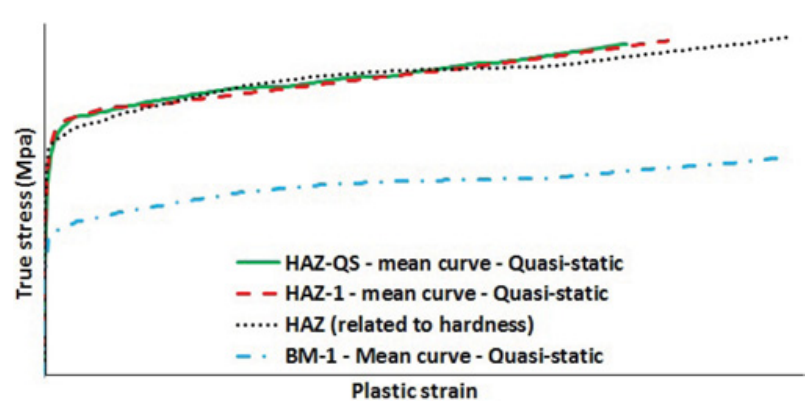

Figure 7. BM and HAZ quasi-static true stress-strain curves.

stresses and obtain the equivalent stress $\left(\sigma_{e q}\right)$. Necking effects are easily quantified for specimens with circular cross-sections using the Bridgman correction approach. In the center of the sample the triaxiality stress ratio is calculated by the relation:

$$
\sigma^{*}=\frac{1}{3}+\ln \left(1+\frac{a}{2 R}\right) .
$$

Where $a$ is the current radius of the neck and $R$ is the radius of curvature of the neck surface in the longitudinal plane at the minimum section. The Bridgman and LeRoy correction ([14] \& [15]) is defined by:

$$
\overline{\sigma_{e q}}=\frac{\sigma_{z z}}{\left(1+\frac{2 R}{a}\right) \ln \left(1+\frac{a}{2 R}\right)} .
$$

LeRoy [15] found that the neck geometry could be calculated with the following approximation and gives good results for steel with $\kappa=1.1$ :

$$
\frac{a}{R}=\kappa\left(\overline{\varepsilon_{p}}-\varepsilon_{u}\right) \text { for } \overline{\varepsilon_{p}}>\varepsilon_{u} .
$$

\section{Experimental results}

In the first part of this section, the BM and HAZ mechanical behaviors are compared thanks to their true stress-strain behavior laws. The strain-rate dependency of each material is investigated in the second part. Those true stress-strain behavior laws are obtained up to failure by using specimen contour evolution by video tracking associated with the Bridgman-LeRoy correction. It can be noted that, across all further analyses, for each test the strain rate is considered to be constant until failure occurs and is equal to the value measured in the gage length before necking. In the neck, the local strain rate increases significantly with the diameter reduction. To access to this strain rate evolution the measurement of strain fields by DIC would be necessary.

\subsection{Quasi-static $B M$ and $H A Z$ tests results}

The quasi-static true stress-strain behaviors for both materials are presented in Fig. 7. The specimen geometries are referred by BM-1, HAZ-QS and HAZ-1 (Fig. 4). Except the experimental scatter at failure, the true stressstrain behavior related to the HAZ with the two different specimen geometries are similar (curves HAZ-1 \& HAZQS in Fig. 7). The Bridgman-LeRoy correction allows to

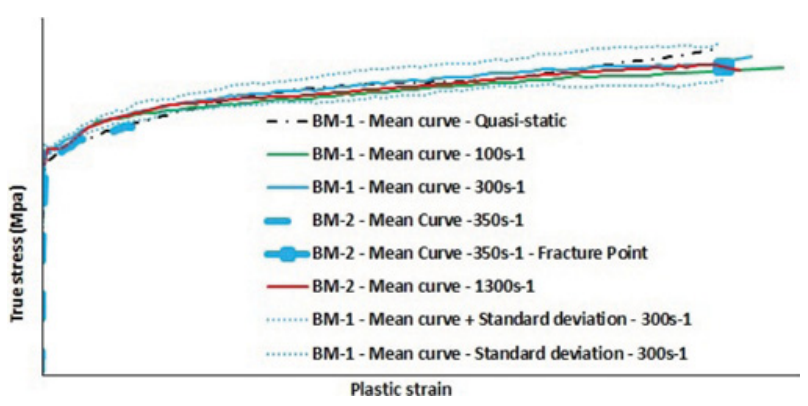

Figure 8. BM - True stress-strain curve - Strain rate dependency.

determine the local material behavior. The microstructure of the MB is a martensitic lath structure which provided ductility and toughness. The heat treatment has created a new martensitic structure with a smaller grain size number. So, the yield stress and ultimate tensile stress have increased on the HAZ material but strain at failure has decreased. In fact, the applied thermal cycle is known to act as a heat treatment which hardens the material.

An alternative way, based on an engineering approximation, allows to calibrate the HAZ behavior law by scaling with a constant factor, the BM behavior law related to the hardness of HAZ material. The obtained HAZ behavior is quite close to the real measured HAZ behavior (Fig. 7).

\subsection{BM strain rate dependency}

In this section, the BM true stress-strain behavior is analyzed for strain rates from the quasi-static range up to $1300 \mathrm{~s}^{-1}$ (Fig. 8). The specimen geometries are referenced by BM-1 and BM-2 which only differ by the gage length (Fig. 4). Only the experimental scatter at $300 \mathrm{~s}^{-1}$ with the BM-1 specimen geometry is presented here. To compare the impact of the specimen geometry, additional tests at $300 \mathrm{~s}^{-1}$ (and $350 \mathrm{~s}^{-1}$ ) for both BM specimen geometries (BM-1 and BM-2) are led. As a video problem appeared during test at $350 \mathrm{~s}^{-1}$ on the BM-2 specimen geometry, only the pre-necking behavior law and the stress-strain point at failure calculated post-mortem are presented. We observe that BM-2 hardening law is contained in the BM-1 experimental scatter at $300 \mathrm{~s}^{-1}$. All mean curves, from quasi-static up to $1300 \mathrm{~s}^{-1}$, are contained in the BM-1 experimental scatter at $300 \mathrm{~s}^{-1}$, so the BM hardening is not strain rate sensitive.

We can conclude that, thanks to the specimen contour tracking and the Bridgman-LeRoy correction, the geometry effects after necking do not seem to impact the BM material behavior or it is drowned in the experimental scatter. Regarding the strain at failure, a reverse engineering approach, based on the engineering stress and strain behavior laws up to failure, is however needed to identify a geometry insensitive failure criterion.

Triaxiality factor for quasi-static and dynamic tests (with the different geometries) are in the order of 0.8 at failure in the center of the specimen. 


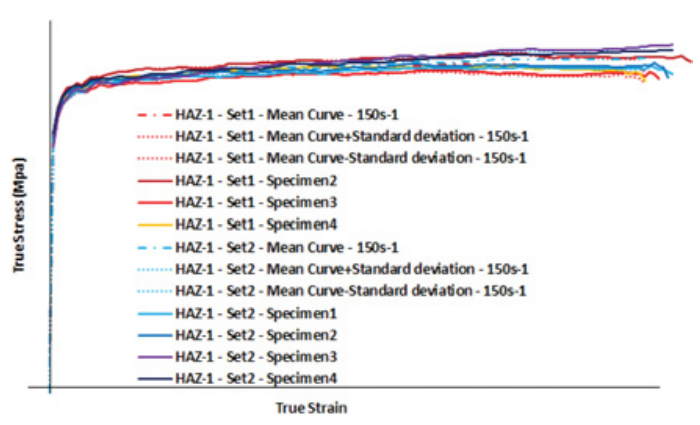

Figure 9. HAZ - True stress-strain curve - Experimental scatter.

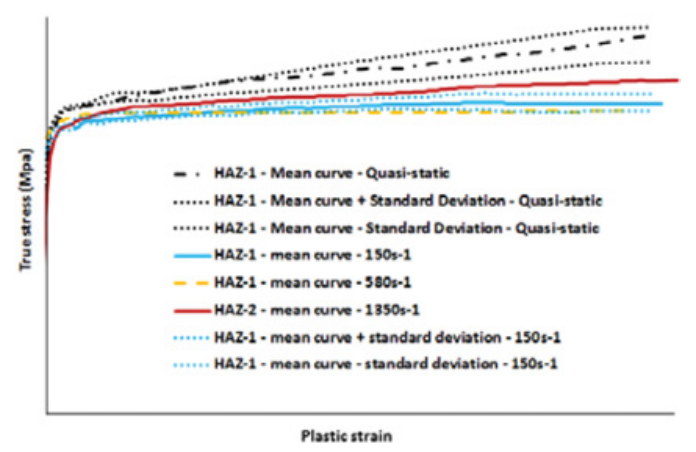

Figure 10. HAZ - True stress-strain curve - Strain rate dependency.

\subsection{HAZ strain rate dependency}

In this last part, the HAZ behavior is studied at different strain rates from quasi-static up to $1350 \mathrm{~s}^{-1}$ (Fig. 10). The specimen geometries are referenced by HAZ-1 and HAZ2 (Fig. 10). To study and validate the repeatability of the applied thermal cycle, two sets of 4 samples are tested with the same strain rate $\left(150 \mathrm{~s}^{-1}\right)$. One set shows more scatter (a relative difference of $7 \%$ on the strain at failure) against $1 \%$ for the other. Nevertheless, the both two mean curves are included in the experimental scatter of the other one (Fig. 9). It can be concluded that thermal cycle is a repeatable operation.

Figure 10 shows, firstly, a softening between quasistatic and dynamic tests results, both on yield stress and stress at failure. Secondly, for dynamic tests, the strain rate dependency between $150 \mathrm{~s}^{-1}$ and $1350 \mathrm{~s}^{-1}$ is not explicit. Indeed, with the HAZ-1 geometry, there is a softening with the increase of the strain rate $\left(150 \mathrm{~s}^{-1}\right.$ and $\left.580 \mathrm{~s}^{-1}\right)$. Tests performed with the HAZ-2 geometry at $1350 \mathrm{~s}^{-1}$ shows a slight hardening by comparison to HAZ-1 dynamic tests results. Experimental scatters could not explain this gap. Stress triaxiality factor are quite similar between all those tests.

At this time we are not able to explain those unexpected results. Regarding the softening, the microstructure and the chemical composition generated by the heat treatment will be deeply analyzed across the tested specimens. Hardness measurements, across a longitudinal section in the postmortem sample, will be also made. Regarding potential geometry effects, quasi-static and dynamic $\left(580 \mathrm{~s}^{-1}\right)$ tests with the HAZ-2 specimen geometry will be led to quantify if geometrical effects impact the HAZ true stress-strain curve.

\section{Conclusion and prospects}

This paper presents the evolution of the behavior between $\mathrm{BM}$ and HAZ materials of a HSS fillet welded joint assembly from quasi-static to dynamic loadings. First, a specific approach is developed to generate the HAZ material using a thermal treatment. Hardness and grain size are used to validate the replicated HAZ material. This approach appears efficient and repeatable. To cover a large range of strain rates, different specimen geometries are needed. A direct method, using video tracking of the specimen geometry and Bridgman-LeRoy correction is used to identify the true stress-strain curve. Secondly, quasi-static and dynamic behaviors from BM and HAZ are compared. The thermal cycle affects significantly the material behavior. Indeed, on the HAZ the hardening is increased whereas strain at failure is decreased. Regarding the strain rate effects, it appears that the BM is insensitive while the HAZ presents a softening between quasistatic and dynamic tests. Further analyses are needed on HAZ sample (hardness, chemical composition and microstructure) to understand this dynamic material softening.

\section{References}

[1] E. Markiewicz, P. Ducrocq, P. Drazetic, G. Haugou, T. Fourmentraux, J.Y. Berard, Int. J. of Materials and Product Technology, 16, 484-509 (2001).

[2] B. Langrand, E. Markiewicz, Int. J. of Impact Engineering, 37-7, 792-805 (2010).

[3] T. Wang, Ph.D. thesis, NTNU, 2006.

[4] F. Labesse-Jied, B. Lebrun, E. Petitpas, J.L. Robert, Proceedings of the 6th Int. Conference on Biaxial/Multiaxial Fatigue and Fracture., Lisbon, 2001, Editor: M. De Freitas, 81-89.

[5] T.K. Chan, R.F.D. Porter Goff, Thin-Walled Structures, 36, 265-287 (2000).

[6] H.G. Hildrum, Ph.D. thesis, NTNU, 2002.

[7] P. Gaudreault, A. Bouamoul, R. Durocher, B. StJean, Proceedings of the 22nd Int. Symposium on Ballistics, Vancouver, BC, Canada, 2005.

[8] B. Gailly, Ph.D. thesis, Mines de Paris, 1996.

[9] P. Chevrier, Ph.D. thesis, Université de Metz, 1998.

[10] C. Albertini, M. Montagnani, Mechanical Properties at High Strain Rates, 21, 22-32 (1974).

[11] H. Kolsky, Stress waves in solids, Dover Publications Inc., New York, 1963.

[12] O.S. Hopperstad, T. Borvik, M. Langseth, K. Labibes, C. Albertini, Eur. J. of Mech. A/Solids, 22, 1-13 (2003).

[13] T. Borvik, O.S. Hopperstad, T. Berstad, Eur. J. of Mech. A/Solids, 22, 15-32 (2003).

[14] P.W. Bridgman, Studies in Large Plastic Flow and Fracture, McGraw-Hill, 1952.

[15] G. Le Roy, J.D. Embury, G. Edward, M.F. Ashby, Acta Metall., 29, 1509-1522 (1981). 